\title{
The Realization of Chinese Herbal Pieces quality Management System Based on Internet of Things Technology
}

\author{
Jiadong XIE, Fang Peipei, Kongfa HU*, Chenjun HU
}

Institute of Information Technology of Nanjing University of Chinese Medicine, Nanjing 210023

Keywords: Chinese herbal pieces; RFID; WSN; Android

\begin{abstract}
With the development of "preventive treatment of disease" in Chinese Medicine and the health care industry, it brings the good opportunity for the development of Chinese herbal medicine industry and leads to a large number of demand. While the rapid expansion of the scale, the inferior pieces has become a chronic illness. The inferior Pieces has become a chronic illness. In this paper, we establish the Chinese herbal pieces quality management system through the technology of RFID, WSN, JSON, intelligent mobile terminal, etc. This technology achieves the control and management of Chinese herbal pieces "from field to of oral". It can realize the control and management of pieces from the original drug plant to production and consumption of the whole process.
\end{abstract}

\section{Introduction}

According to statistics, January to October in 2011, Chinese herbal pieces manufacturing industry output value of 68.2 billion yuan, a year-on-year growth of $53.58 \%$. The first three quarters of 2012, according to the statistics of the Ministry of industry and information technology, Chinese herbal pieces was 69.2 billion yuan in output value, growing $27.1 \%$ compared to the same period. At the same time, with the development of "preventive treatment of disease" in Chinese Medicine and the health care industry, it brings the good opportunity for the development of Chinese herbal medicine industry and leads to a large number of demand. While the rapid expansion of the scale, the inferior pieces has become a chronic illness. People's network in March 21, 2013 issued one article "starch sell the 'gold price' and counterfeit also draw near 'famous brand' ", it pointed out that the fraud has staggering profits in the field of Chinese medicine, the fraud means of Chinese herbal medicine constantly update, some fake was unexpectedly difficult to distinguish true from false. For example, flour, corn flour, starch and other materials are processed through the use of a special "Cordyceps mold" after high temperature and pressure, then paint and dry, thus the cases that no medical value of food pretend genuine "Cordyceps" are often seen. Some experts inside course of study points out, we should control the quality of traditional Chinese medicine from the plant source because the Chinese herbal medicine planting is dispersed and Chinese herbal medicine fraud phenomenon does not cure.

In recent years, some researchers have applied RFID technology in the drug supply chain management and drug supervision [1], but the application of RFID in Chinese herbal medicine production management is considerably less and a mature application mode has not yet formed.

In this paper, RFID was applied in production management [2] of Chinese herbal medicine, and the article put forward model of RFID in pieces of Chinese medicine industry, combined with WSN[3], intelligent mobile technology[4] and other technology. The quality management system in Chinese herbal medicine can be established to realize the control and management of pieces from the original drug plant to production and consumption of the whole process.

\footnotetext{
${ }^{*}$ Corresponding author: Kongfa HU

Institute of Information Technology of Nanjing University of Chinese Medicine, Nanjing 210023,

Ph:+862585811570, e-mail: kfhu@njutcm.edu.cn
} 


\section{Related Technology}

Java EE also called as J2EE, the Java Platform Enterprise Edition, is a standard platform of Sun company launched for the enterprise application. Now, J2EE not only refers to a standard platform, it is more an expression of a software architecture and design ideas.

Struts2 is a new MVC framework based on WebWork technology, with WebWork as the core, using interceptor mechanism to handle the user's request, making the business logic controller and Servlet API completely separate and simplify the development cycle of the whole application from the compilation, deployment and maintenance.

Spring is designed to address the complexity of enterprise application development, which can help the development team to build the simple, fast and flexible system and application based on JVM.

Hibernate4.3 is an object relational mapping framework that open source code, it is very lightweight object encapsulation of JDBC, making Java programmers can be free to use object-oriented programming ideas to manipulate the database.

JSON(JavaScript Object Notation) is a lightweight data interchange format, easy to read and write, easy to parse and generate. JSON uses a completely language-independent text format, and uses a similar to the $\mathrm{C}$ language family habits (including C, C++, C\#, Java, JavaScript, Perl, Python, etc. ). These properties make JSON be an ideal data exchange language.

Intelligent mobile terminal refers to the computer equipments which can be used in mobile, including mobile phone, notebook computer, tablet computer, the POS machine, vehicle mounted computer, etc..

Android is a semi open source operating system based on Linux, mainly used in mobile equipment, continuously led and developed by Open Handset Alliance founded by Google.

\section{The Technical Route}

\section{The application model of RFID.}

This system uses RFID tag as the carrier of information, which is a bond jointing planting, production, storage, circulation and supervision of Chinese herbal medicine. From planting, the RFID tags are installed in medicine fields to store the information in the planting process. While entering the warehouse, the raw materials are given RFID tag, so as to establish identity for pieces of the raw materials. The RFID issuing department according to the production plan distributes radio frequency label and associates with production plan, establishes the identity of pieces in the production process. We can deploy the RFID information collection equipment at the place of the production workshop and warehouse, and the hand-held reader in those place can read and write the RFID data, which can be integrated into the quality management system of Chinese herbal pieces. Meanwhile, the RFID combined with the "drug electronic supervision code printing specification" begin to implement in 2010, fully consider the pieces of Chinese medicine enterprise in each link from planting, production, storage and sale of specific application requirements, and build application mode of RFID in Chinese herbal medicine industry.

\section{WSN.}

The paper presents to apply the temperature, humidity, $\mathrm{pH}$ and other sensors in the Chinese herbal medicine planting and its storage. The values of monitoring can be uploaded to the server every 30 seconds, monitor the changes of growth environment of Chinese herbal medicine and storage environment of Chinese herbal pieces, and feed back the parameter values obtained by the sensors in line graph to management personnel. When the monitoring values vary in a large range and are beyond the warning threshold, the system will take the initiative to the PC client system and mobile terminal system push the early warning information, and remind manages to handle.

\section{Crossing platform data exchange.}

This system uses lightweight JavaEE[5] ( Struts2+Spring+Hibernate ) technology to realize the background data encapsulation, and uses the typical JavaEE three-layer architecture, divided into 
the presentation layer, middle layer and data service layer. The three-layer system processes the business rules, data access and verifies the legitimacy of such work on the middle layer. The client does not directly interact with the database, but builds connection with the middle layer through a component, then the middle layer interacts with database, as shown in Figure 1.

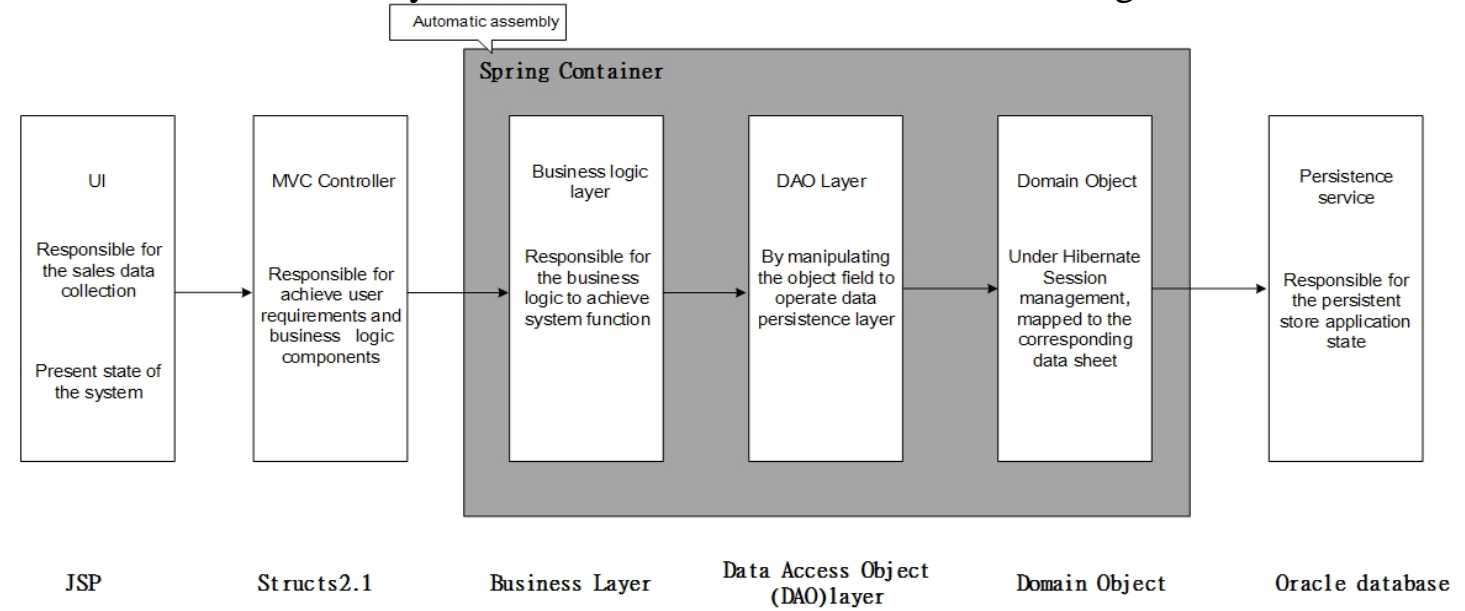

Figure 1. The back-end JavaEE system structure diagram

In the MVC model of Struts, Action instead of Servlet plays the role of Model. For Struts, JSON data back to the client, as with traditional WEB applications. There are two ways, namely the output of JSON data in Action and the output of JSON data in view source. This paper adopts the first method to obtain JSON data, by serializing data and deserializing in order to achieve crossing platform data exchange, thus completing the upload and download data, as show in Figure 2.

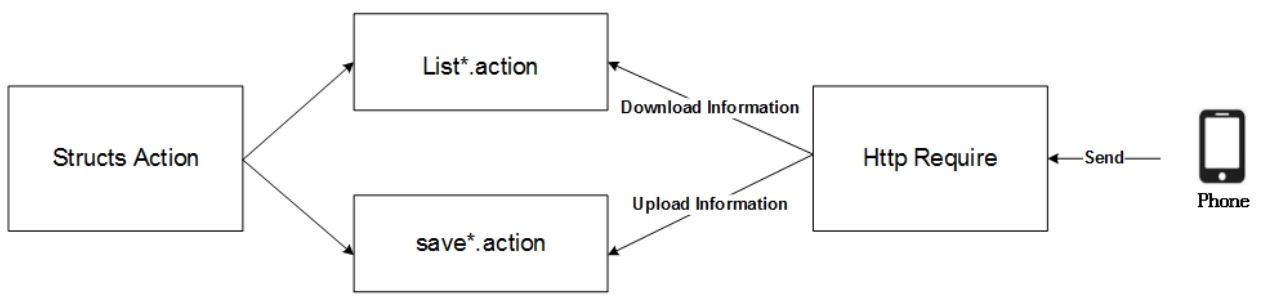

Figure 2. Crossing platform data interaction diagram

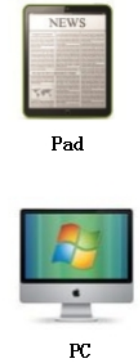

$\mathrm{PC}$

\section{JSON specification.}

Although XML has the advantages of crossing platform and crossing language, however, in the application of Web in general, the developers often break mind for the analysis of XML, because the server generation or processing of XML or client using JavaScript to analyse XML are often leads to complex code, reduces the efficiency of the development. Compared with XML, JSON provides another data exchange format for Web application developers, and provides better simplicity and flexibility.

The system settings of JSON data format is mainly to provide unified data interface for the outside. When they want to get pieces of information, JSON data provided by pieces of production information server program can be analyzed through our authorization, so as to obtain the related information about pieces in the whole production chain.

We set the format of JSON datagram using getListJson() method in the Action, through Struts intercepts and processes user's requests, and returns the required data message.

JSON data format of the final settings is as follows: \{"total": "1", "page": "1", "records": "9", "rows": [\{"key1":"value1","key2":"value2"\}]\}. Where "total" represents the total number of records, "page" for paging query, represents the first few pages, "records" represents that the current page has several records, "rows" represents that the current page shown up to many of the records.

\section{Intelligent mobile terminal.}

The intelligent mobile platform based on Android mobile client[6] develops bar code identification procedures of Chinese herbal medicine, then the procedures scan herbal pieces of electronic monitoring code, based on the recognized bar code information, online query the whole 
process of production of Chinese herbal pieces of information.

This platform uses development interface provided by Google Zxing to develop the drug electronic supervision code in order to scan and recognize application. The system using HTTP protocol sends recognized supervision code information in the form of Get to the server and sends requests, and obtains the medicines information associated with the electronic supervision code.

\section{Realization of the system functions}

This system adopts the three-layer architecture design patterns, respectively, the data service layer, the business logic layer and the presentation layer. The data service layer mainly interacts with Oracle through Hibernate, and provides business logic layer with the main pieces of the corresponding DAO. The business logic layer achieves automatic assembly with the presentation layer Struts through Spring transaction and AOP section programming. The presentation layer provides the interaction of Struts, JSP and jQuery to realize the system front-end display.

Combining Struts, Spring and Hibernate with each other, we constitute the three-layer architecture of system background applications, and provide the data interface, than it can interact for other system application platform via the action.

Hibernate implements the data access layer via encapsulating JDBC and the realization of the code are as following:

$<$ bean id="dataSource" class="org.apache.commons.dbcp.BasicDataSource" $>$

$<$ property name $=$ "driverClassName" value="oracle.jdbc.OracleDriver" $></$ property $>$

<property name="url" value="jdbc:oracle:thin:@localhost:1521:orcl" $></$ property $>$

$<$ property name="username" value="system" $><$ property $>$

$<$ property name="password" value="passwd" $><$ property $>$

\section{$<$ /bean $>$}

Integrated Hibernate and Spring with the aid of the Spring configuration to create a SessionFactory object, this paper uses the LocalSessionFactoryBean and uses the programming method interior completely to configure the configuration object, and access sessionFactory. The realization of the code are as following:

$<$ bean id="sessionFactory" class="org.springframework.orm.hibernate3.LocalSessionFactoryBean">

$<$ property name="dataSource" $><$ ref bean="dataSource" / $><$ property $>$

$<$ property name="mappingDirectoryLocations" $>$

$<$ list $><$ value $>$ WEB-INF/classes/com/njutcm/qsmes/Domain/hbm $</$ value $></$ list $>$

$<$ property $>$

$<$ property name="hibernateProperties" $>$

$<$ props $>$

$<$ prop key="hibernate.hbm2ddl.auto" $>$ update $<$ prop $>$

$<$ prop key="hibernate.dialect" $>$ org.hibernate.dialect.Oracle10gDialect $</$ prop $>$

$</$ props $>$

$</$ property $>$

$<$ /bean $>$

Integrated Spring and Struts, and configured Action of Struts as a JavaBean of Spring, it only need to define the corresponding variables and the setter/getter method of variables, in this way, which can pour into an object for it via Spring, the code as follows:

$<$ bean id="PackDetailRecAction" class="com.njutcm.qsmes.Action.PackDetailRecAction" scope="prototype">

$<$ property name="baseService" ref="baseService" $>>$

$<$ property name="keyBaseService" ref="baseService" $>$

$<$ /bean $>$

By intercepting each platform to the back-end initiated requests, and serializing or deserializing the request parameters, Struts provide JSON data interface crossing platform for each platform. The Struts configuration is as following: 
<action name="listDictAccessory" class="DictAccessoryAction" method="list">

$<$ result name="success" type="json" $></$ result $>$

$</$ action $>$

The above-mentioned Struts configuration has integrated Spring, if it does not integrate Spring, the class attribute value in the configuration should be the qualified name of global class, namely the package name plus class name. Struts corresponds to the getListJson() method in action class to achieve Java Object serialized as JSON object with the help of net.sf.json.JSONArray or net.sf.json.JSONObjec etc. The specific implementation methods are as following:

List $<$ EntityClass $>$ list $=$ new ArrayList $<$ EntityClass $>()$;

for (; instance.hasNext(); $\{\quad$ list.add(instance.next()); $\}$

String json = JSONArray.fromObject(list ,jsonConfig).toString();

jsonBuilder.append(json);

Finally, we create the extended ServletRequest interface HttpServletResponse, providing specific sending and response functions for HTTP. The configuration is as following:

HttpServletResponse response $=$ ServletActionContext.getResponse();

response.setContentType("application/json");

response.setCharacterEncoding("UTF-8");

response.getWriter().write(jsonBuilder.toString());

Chinese herbal medicine information tracing.

By scanning the drug electronic supervision code, Intelligent mobile terminal submits a HTTP request to query and obtain pieces of Chinese medicine "from field to verbal" information. The specific implementation details are as follows:

First of all, it defines a HttpUtils tools class, and creates a method getRequest for Get request to send a request to the server program. The realization of the code are as following:

public static String getRequest(String uri)\{

String result $=$ null;

HttpGet httpRequest = new HttpGet(uri);

try \{

HttpResponse httpResponse = new DefaultHttpClient(new BasicHttpParams()).execute(httpRequest); result $=($ httpResponse.getStatusLine ()$\cdot$ getStatusCode ()$==200)$ ? EntityUtils.toString(httpResponse .getEntity()) : "Bad Request!";

\} catch (Exception e) \{ $\quad$ e.printStackTrace(); $\quad$ result = "network refused!"; $\quad\}$

return result;

\}

Requesting the above method will obtain the Chinese herbal medicine information containing JSON string, then call the getObjectLists method, using GsonBuilder to generate Gson object and stipulating the contents of Gson serialization and deserialization format. The JSON string is transformed into Java Object. The getObjectLists method is implemented as following: public static List $<$ MakeDuty $>$ getObjectLists(String json) \{

List $<$ MakeDuty $>$ lists $=$ new ArrayList $<$ MakeDuty $>()$;

try \{

int a $=$ json.indexOf("[") ;//canned format

json = json.substring(a, json.length() - 1);

GsonBuilder gsonBuilder = new GsonBuilder(); //gsonBuilder.setDateFormat("yyyy-MM-dd hh:mm "); gsonBuilder.registerTypeAdapter(Timestamp.class,new TimestampTypeAdapter());

Gson GSON = gsonBuilder.create();

lists = GSON.fromJson(json, new TypeToken $<$ List $<$ MakeDuty $>>()\{\}$.getType()); // json convert to list \} catch (JsonSyntaxException e) \{ e.printStackTrace(); \}

return lists; 
Finally, Java Object List acquired by TabHost is displayed on the system interface for the user to pieces quality tracking.

\section{Summary}

In this paper, the quality management system of Chinese herbal pieces was established based on the RFID, WSN, JavaEE, JSON and intelligent mobile terminal technology. Firstly, we establish the application mode of RFID in the Chinese herbal medicine industry, convenient for management and traceability of pieces in production and processing as well as after sales. Secondly, we apply the sensors in Chinese herbal medicine planting and pieces storage link, monitoring the field and warehouse environment change in real time to ensure the quality and quantity of the medicinal materials. Finally, we analyze and introduce how to use the lightweight JavaEE to integrate back-end system so as to provide the interface of the data interaction crossing platform for other application platform, JSON interface specification. We describe the Android platform called JSON interface in order to implement the quality management and traceability of Chinese herbal pieces "from field to oral".

\section{Acknowledgement}

The research in the paper is supported by the Cooperative Innovation Fund of Jiangsu Province of China under Grant No. BY2013063-08; the Science and Technology Support Fund of Jiangsu Province, China Grant No. BE2012184; the "333 Project” of Jiangsu Province of China under Grant No. BRA2012156; the "Six Talent Peaks Program” of Jiangsu Province of China under Grant No. 2009180; the Natural Science Foundation of Jiangsu Province,China (BK20140958).

\section{References}

[1] TAN Hui. Drug Supply Chain Management System Based on RFID Technology [J]. China Manufacturing Informatization,2011,21:17-20+25.

[2] TANG Heng. Planning and Design of Drug Tracing Management System Based on RFID Technology [D].Dalian University of Technology,2013.

[3] YU Liang, WANG Zhibo, LUO Ji'an, etc.. Wireless Sensor Network QoS Design of Index System for Moving Target Tracking [J]. Chines Journal of Computers,2009,03:441-462.

[4] CAI Weihong, OU Hongyu, LI Ruyin. Research on Intelligent Mobile Terminal Technology Development Impact on Mobile Learning in Environment of Three Nets Fusion [J]. Journal of Changsha Tel \& Tec Vocational College,2012,04:1-4.

[5] GE Hanqiang. Research and Implementation of lightweight Web Application Framework Based on JavaEE. [D].Wuhan University of Science and Technology,2008.

[6] GONG Chengying, XING Jinghong, HU Yinbao. Android Mobile Terminal and PHP and MySQL Data Communication Based on JSON [J]. Industrial Instrumentation and Automation Device,2013,01:63-65+69. 